\title{
Universiteit
}

Leiden

The Netherlands

\section{Orbital angular momentum analysis of high-dimensional entanglement} Peeters, W.H.; Verstegen, E.J.K.; Exter, M.P. van

\section{Citation}

Peeters, W. H., Verstegen, E. J. K., \& Exter, M. P. van. (2007). Orbital angular momentum analysis of high-dimensional entanglement. Physical Review A, 76, 042302.

doi:10.1103/PhysRevA.76.042302

Version: $\quad$ Not Applicable (or Unknown)

License: $\quad$ Leiden University Non-exclusive license

Downloaded from: https://hdl.handle.net/1887/61294

Note: To cite this publication please use the final published version (if applicable). 


\title{
Orbital angular momentum analysis of high-dimensional entanglement
}

\author{
W. H. Peeters, ${ }^{1}$ E. J. K. Verstegen, ${ }^{2}$ and M. P. van Exter $^{1}$ \\ ${ }^{1}$ Leiden University, Huygens Laboratory, P.O. Box 9504, 2300 RA Leiden, The Netherlands \\ ${ }^{2}$ Philips Research Laboratories, High Tech Campus 11, 5656 AE Eindhoven, The Netherlands
}

(Received 2 March 2007; published 1 October 2007)

\begin{abstract}
We describe a simple experiment that is ideally suited to analyze the high-dimensional entanglement contained in the orbital angular momenta (OAM) of entangled photon pairs. For this purpose we use a two-photon interferometer with a built-in image rotator and measure the two-photon visibility versus rotation angle. Mode selection with apertures allows one to tune the dimensionality of the entanglement; mode selection with spiral phase plates and fibers allows detection of a single OAM mode. The experiment is analyzed in two different ways: either via the continuous two-photon amplitude function or via a discrete modal (Schmidt) decomposition of this function. The latter approach proves to be very fruitful, as it provides a complete characterization of the OAM entanglement.
\end{abstract}

DOI: 10.1103/PhysRevA.76.042302

PACS number(s): 03.67.Mn, 42.50.Dv, 42.65.Lm

\section{INTRODUCTION}

Spontaneous parametric down-conversion (SPDC), in which a pump photon splits into two photons of lower energy, is a common technique to produce quantum-entangled photon pairs [1-4]. The generated photon pairs can be entangled in three degrees of freedom. The best-studied form of entanglement is that of the polarization [2], which spans a two-dimensional space and can thus be described in terms of qubits. The two other forms of entanglement involve either the time-frequency entanglement or the position-momentum entanglement within the photon pair. As these forms of entanglement involve continuous variables, the states are contained in a space of much higher dimension and described by qunits instead of qubits. One of the first experiments on timefrequency entanglement was the two-photon interference experiment of Hong, Ou, and Mandel [1], who demonstrated photon bunching at equal arrival times. Other forms of timefrequency entanglement have recently been studied by Gisin et al. [3].

In this paper, we will discuss the nature of spatial entanglement, where a measurement on the position-momentum of one photon fixes the spatial profile of the other. This form of entanglement is rapidly attracting more attention [4-9]. We will separate the spatial profiles in radial and azimuthal components and concentrate on the azimuthal part, which can be described in terms of the photon's orbital angular momentum (OAM).

The questions that we will address both theoretically and experimentally deal with the nature of the spatial entanglement: "How many modes are involved in the spatial entanglement?" "What is the profile of these spatial eigenmodes?," "How can we separate the radial and azimuthal components?" and "What is the intensity distribution over the orbital angular momentum (OAM) modes and the related Schmidt number?" For our experimental analysis of the nature of the OAM entanglement, we will use a two-photon interferometer with an odd number of mirrors and an image rotator in one of its arms. A measurement of the two-photon interference as a function of the rotation angle proves to be sufficient for a full characterization of the OAM entangle- ment. This paper addresses the theory and confirms and extends earlier experimental results from our group [10].

This paper is organized as follows. In Secs. II and III we present two different theoretical descriptions of the interference in a two-photon Hong-Ou-Mandel (HOM) interferometer with a built-in rotator. The first analysis is based on a continuous representation of the two-photon amplitude function $A\left(\mathbf{x}_{1}, \mathbf{x}_{2}\right)$. The second analysis uses a modal decomposition of the detected two-photon amplitude into a discrete set of eigenmodes. This analysis yields an important and intuitively simple expression for the angle-dependent twophoton interference as a Fourier series over the OAM eigenmodes. In Sec. IV, we present our setup and the obtained experimental results. We demonstrate that our method allows for a full characterization of the entanglement in orbital angular momentum. We apply this method both to a spatially filtered beam and a single-mode beam with a fixed OAM. We end with a concluding discussion in Sec. V.

\section{CONTINUOUS TWO-PHOTON AMPLITUDE}

\section{A. Generated two-photon amplitude}

The two-photon amplitude that is generated in spontaneous parametric down-conversion (SPDC) is relatively simple in the quasimonochromatic paraxial thin-crystal limit, which applies to our experiment. We use a $\mathrm{cw}$ monochromatic pump (at optical frequency $\omega_{p}$ ) with perfect spatial coherence and consider almost frequency-degenerate SPDC, where both photons have approximately the same frequency $\omega_{0} \equiv \omega_{p} / 2$. We operate in the paraxial regime, with generated beams close to the direction of the pump beam. Finally, we take care to operate in the thin-crystal limit, where phase matching is well satisfied within the narrow spectral bandwidth and limited spatial extent of the detection system [11].

In the thin-crystal limit, the generated two-photon field amplitude is [12]

$$
A_{g}\left(\mathbf{r}_{s}, \mathbf{r}_{i}\right) \propto \int h\left(\mathbf{r}_{s}, \mathbf{x}\right) h\left(\mathbf{r}_{i}, \mathbf{x}\right) E_{p}(\mathbf{x}) d \mathbf{x},
$$

where $E_{p}(\mathbf{x})$ is the field profile of the pump beam at the crystal $(z=0)$ with transverse coordinate $\mathbf{x}$. The three dimen- 


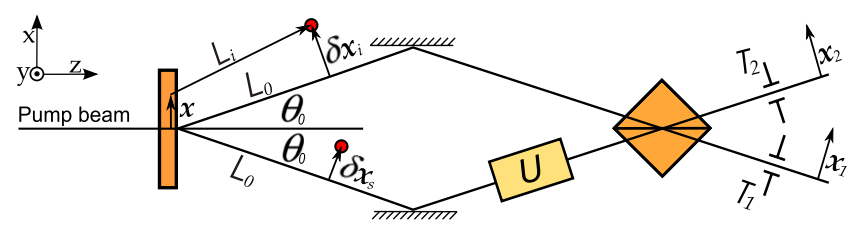

FIG. 1. (Color online) Sketch of a two-photon interferometer containing a built-in image transformation $U$ and the definition of various "beam coordinates." We use the following transverse coordinates: $\delta \mathbf{x}_{s}$ and $\delta \mathbf{x}_{i}$ for the relative positions within the signal and idler beams, and $\mathbf{x}_{1}$ and $\mathbf{x}_{2}$ for the positions at the detectors, again with respect to fixed beam lines. $L_{0}$ is the distance along a fixed beam line from the crystal to the detection planes. The photon propagation lengths $L_{i, s}$ and the transverse position on the crystal $\mathbf{x}$ are used in the integrand of Eq. (1) to calculate the two-photon amplitude.

sional vectors $\mathbf{r}_{s}$ and $\mathbf{r}_{i}$ are the coordinates of the two-photon amplitude. The one-photon propagators $h\left(\mathbf{r}_{s, i}, \mathbf{x}\right)=k_{s, i}$ / $\left(2 \pi i L_{s, i}\right) \exp \left(i k_{s, i} L_{s, i}\right)$ describe free space propagation of either signal or idler photon from the crystal to the detector, where $k_{s, i} \equiv \omega_{s, i} / c$ are their wave numbers (obeying $\omega_{s}+\omega_{i}$ $\left.=\omega_{p}\right)$ and $L_{s, i}=\left|\mathbf{r}_{s, i}-(\mathbf{x}, 0)\right|$ their path lengths.

In the quasimonochromatic paraxial thin-crystal limit, one can express the generated two-photon amplitude in terms of the pump field behind the crystal. The precise form of this relation depends on the chosen coordinate system $[13,14]$. For noncollinear SPDC, we will use "beam coordinates" in which the signal and idler coordinates of the generated twophoton amplitude are defined with respect to two fixed beam axes pointing in the $-\theta_{0}$ and $+\theta_{0}$ directions, respectively. We use $\delta \mathbf{x}_{s, i}$ for the transverse coordinates of the signal and idler photon and $L_{0}$ for the propagation length along each beam axis (see Fig. 1). In the paraxial limit $\left(\theta_{0} \ll 1\right.$ and $|\mathbf{x}|,\left|\delta \mathbf{x}_{s, i}\right|$ $\left.\ll L_{0}\right)$ the photon propagation lengths $L_{s, i}$ become

$$
L_{s, i}=L_{0} \pm x_{x} \theta_{0}+\frac{\left|\delta \mathbf{x}_{s, i}-\mathbf{x}\right|^{2}}{2 L_{0}},
$$

where $x_{x}$ is the $x$ component of $\mathbf{x}$, which is the component in the plane defined by the $z$ axis and the two beam axes. Finally, also working in the quasimonochromatic limit $\left(\mid k_{s}\right.$ $\left.-k_{i} \mid \ll k_{p}\right)$, the generated two-photon amplitude of Eq. (1) becomes

$$
A_{g}\left(\delta \mathbf{x}_{s}, \delta \mathbf{x}_{i}, L_{0}\right) \propto \frac{E_{z}\left(\frac{1}{2}\left(\delta \mathbf{x}_{s}+\delta \mathbf{x}_{i}\right)\right)}{L_{0}} \exp \left(\frac{i k_{p}}{8 L_{0}}\left|\delta \mathbf{x}_{s}-\delta \mathbf{x}_{i}\right|^{2}\right),
$$

where $E_{z}(\mathbf{x})$ is the pump profile in the transverse plane at a distance $z=L_{0}$ behind the crystal [14]. The advantage of beam coordinates in comparison with Cartesian coordinates is that the phase factor relating $E_{z}$ and $A_{g}$ is much smaller in beam coordinates. Equation (3) shows that the generated two-photon amplitude is not only invariant under permutation of the Cartesian coordinates $\mathbf{r}_{s} \leftrightarrow \mathbf{r}_{i}$, but even remains unchanged under permutation of the local beam coordinates $\delta \mathbf{x}_{s} \leftrightarrow \delta \mathbf{x}_{i}$

\section{B. Interference after image rotation}

In this subsection we will first present a theoretical description of a two-photon interferometer with an image transformation $U$ in one of its arms (see Fig. 1). An image transformation $U$ acts as a coordinate transformation of the form $E_{\text {out }}\left(\mathbf{x}_{\text {out }}\right)=E_{\text {out }}\left(U \mathbf{x}_{\text {in }}\right)=E_{\text {in }}\left(\mathbf{x}_{\text {in }}\right)=E_{\text {in }}\left(U^{-1} \mathbf{x}_{\text {out }}\right)$. We will then derive an expression for the two-photon bunching visibility, assuming $U$ to be an orthogonal matrix (comprising image rotations and reflections) and the pump beam to be rotationally symmetric. In the final part we will focus on the important experimental case of an interferometer with an odd number of mirrors and an image rotator, as only this interferometer allows for a characterization of the OAM entanglement (see below).

In order to calculate the detected coincidence rate, we need to express the two-photon amplitude at the detectors $A_{12}\left(\mathbf{x}_{1}, \mathbf{x}_{2}\right)$ in terms of the generated field $A_{g}\left(\delta \mathbf{x}_{s}, \delta \mathbf{x}_{i}\right)$. We do so by accumulating all image operations for the two relevant propagation channels, being the "double transmission" and "double reflection" of the incident photon pair at the beam splitter. For the double transmission channel, these operations are $\mathbf{x}_{2}=U M_{y} \mathbf{x}_{s}$ and $\mathbf{x}_{1}=M_{y} \mathbf{x}_{i}$, whereas the double reflection channel corresponds to $\mathbf{x}_{1}=M_{y} U M_{y} \mathbf{x}_{s}$ and $\mathbf{x}_{2}=\mathbf{x}_{i}$. Here, the operations $M_{y}$ arise from reflections on the mirrors and beam splitter in the interferometer. The two-photon amplitude at the detectors thus becomes

$$
\begin{aligned}
A_{12}\left(\mathbf{x}_{1}, \mathbf{x}_{2} ; \Delta \omega\right)= & T_{\mathrm{bs}} e^{-i(1 / 2) \Delta \omega \tau} A_{g}\left(M_{y} U^{-1} \mathbf{x}_{2}, M_{y} \mathbf{x}_{1}\right) \\
& -R_{\mathrm{bs}} e^{i(1 / 2) \Delta \omega \tau} A_{g}\left(M_{y} U^{-1} M_{y} \mathbf{x}_{1}, \mathbf{x}_{2}\right),
\end{aligned}
$$

where $\Delta \omega \equiv \omega_{1}-\omega_{2}$ is the frequency difference between photons 1 and 2 and $\tau \equiv\left(L_{s}-L_{i}\right) / c$ is the time delay difference in the interferometer. $T_{\mathrm{bs}}$ and $R_{\mathrm{bs}}$ are the intensity transmission and reflection coefficients of the beam splitter, which can also be written as $T_{\mathrm{bs}}=t^{2}$ and $-R_{\mathrm{bs}}=(i r)^{2}$, where $t$ and $r$ are the real-valued amplitude transmission and reflection coefficients of the beam splitter. We will assume the beam splitter to be balanced at $T_{\mathrm{bs}}=R_{\mathrm{bs}}=\frac{1}{2}$. The coincidence rate for simultaneous photon detection with large (bucket) detectors behind two apertures with transmission profiles $T_{1}\left(\mathbf{x}_{1}\right)$ and $T_{2}\left(\mathbf{x}_{2}\right)$ is obtained after spatial and spectral integration via

$$
\begin{aligned}
R_{\mathrm{cc}}(\tau) \propto & \iiint\left|A_{12}\left(\mathbf{x}_{1}, \mathbf{x}_{2} ; \Delta \omega\right)\right|^{2} T_{1}\left(\mathbf{x}_{1}\right) \\
& \times T_{2}\left(\mathbf{x}_{2}\right) T_{\mathrm{tot}}(\Delta \omega) d \mathbf{x}_{1} d \mathbf{x}_{2} d \Delta \omega,
\end{aligned}
$$

where $T_{\text {tot }}(\Delta \omega) \equiv T_{f 1}\left(\omega_{0}+\frac{1}{2} \Delta \omega\right) T_{f 2}\left(\omega_{0}-\frac{1}{2} \Delta \omega\right)$ and $T_{f 1}(\omega)$ and $T_{f 2}(\omega)$ are the intensity transmission spectra of the bandpass filters situated in front of detectors 1 and 2 .

Two-photon interference can best be observed by measuring the coincidence rate $R_{\mathrm{cc}}$ as a function of the time delay $\tau$ experienced in the interferometer. We distinguish two extreme cases for the time delay: $\tau=\infty$, where interference is absent, and $\tau=0$, where the interference is strong and where one generally observes a so-called Hong-Ou-Mandel (HOM) $\operatorname{dip}[1]$ in the coincidence rate $R_{\mathrm{cc}}(\tau)$. We quantify the strength of the two-photon interference, i.e., the depth of HOM dip, by defining the two-photon bunching visibility as 


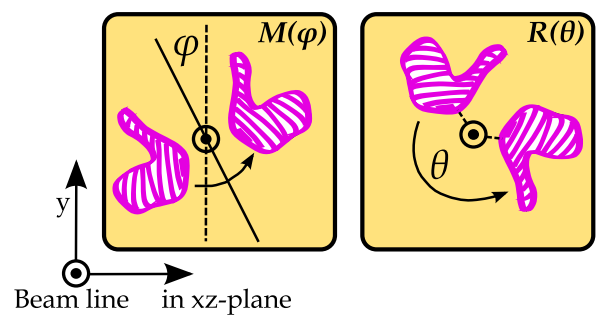

FIG. 2. (Color online) Graphical representation of the two generic orthogonal image transformations $M(\phi)$ and $R(\theta)$ in a plane orthogonal to a beam line. The beam line is pointing out of the paper. $M(\phi)$ is a reflection in a line making an angle $\phi$ with the $y$ axis. $R(\theta)$ is a rotation of an angle $\theta$ around the beam line.

$$
V \equiv 1-\frac{R_{\mathrm{cc}}(\tau=0)}{R_{\mathrm{cc}}(\tau=\infty)} .
$$

The two-photon bunching visibility of our interferometer with built-in image transformation $U$ (see Fig. 1) can now be calculated by combining Eqs. (3)-(6). In order to simplify the final expression we will restrict our analysis in three ways. First, we assume the pump field to be rotationallysymmetric with zero orbital angular momentum. Second, we consider only orthogonal image transformations which comprise any combination of image reflections and image rotations (see Fig. 2). We define $M(\phi)$ as an image reflection in a line oriented at an angle $\phi$ with respect to the $y$ axis, and $R(\theta)$ as an image rotation over an angle $\theta$. Finally, we assume one aperture to be fully open, i.e., $T_{1}\left(\mathbf{x}_{1}\right)=1$.

The first two restrictions allow us to combine all image operations into a single matrix $U_{\text {tot }}=U M_{y} U M_{y}$ and reduce the two-photon amplitudes in Eq. (4) to $A_{g}\left(\mathbf{x}_{1}^{\prime}, \mathbf{x}_{2}\right)$ and $A_{g}\left(\mathbf{x}_{1}^{\prime}, U_{\text {tot }} \mathbf{x}_{2}\right)$. The third restriction allows us to isolate the transverse correlation function of the pump field. The expression for the two-photon bunching visibility thus becomes

$$
V(\theta)=\frac{\int \widetilde{g}_{z}\left[\frac{1}{2}\left(U_{\text {tot }}-1\right) \mathbf{x}_{2}\right] T_{2}\left(\mathbf{x}_{2}\right) d \mathbf{x}_{2}}{\int \widetilde{g}_{z}(\mathbf{0}) T_{2}\left(\mathbf{x}_{2}\right) d \mathbf{x}_{2}},
$$

where the normalized correlation function of the pump field (in spherical coordinates) is defined as

$$
\widetilde{g}_{z}(\delta \mathbf{x})=\frac{\int \widetilde{E}_{z}^{*}\left(\mathbf{x}+\frac{1}{2} \delta \mathbf{x}\right) \widetilde{E}_{z}\left(\mathbf{x}-\frac{1}{2} \delta \mathbf{x}\right) d \mathbf{x}}{\int\left|\widetilde{E}_{z}(\mathbf{x})\right|^{2} d \mathbf{x}} .
$$

This function is always real-valued due to the symmetry of the pump. The pump field $\widetilde{E}_{z}(\mathbf{x})$ in spherical coordinates is related to the pump field in Cartesian coordinates as

$$
\widetilde{E}_{z}(\mathbf{x}) \equiv E_{z}(\mathbf{x}) \exp \left(-\frac{i k_{p}}{2 z}|\mathbf{x}|^{2}\right) .
$$

Note that we have incorporated all phase factors in $\widetilde{E}_{z}(\mathbf{x})$ by choosing a convenient spherical coordinate system that has its origin at the center of the pump spot on the crystal. The pump profile $\widetilde{E}_{z}(\mathbf{x})$ becomes real-valued in the far field, but is complex in the near field. As a result, only the far-field correlation function is directly related to the intensity profile of the pump in the detection plane. The near-field correlation function on the other hand is much narrower than the pump profile in the corresponding plane. For the experimentally important case of a Gaussian TEM $_{00}$ pump that is mildly focused at the crystal as $E_{0}(\mathbf{x}) \propto \exp \left(-|\mathbf{x}|^{2} / w_{0}^{2}\right)$, this correlation function is

$$
\widetilde{g}_{z}(\mathbf{x}) \propto \exp \left[-\frac{|\mathbf{x}|^{2}}{2 w_{z}^{2}}\left(1+\frac{z_{0}^{2}}{z^{2}}\right)\right],
$$

where $w_{z}=w_{0} \sqrt{1+\left(z / z_{0}\right)^{2}}$ is the width of the pump beam in the detection plane and $z_{0} \equiv \frac{1}{2} w_{0}^{2} k_{p}$ is the Rayleigh range of the pump beam.

There are two distinct possibilities for the orthogonal matrix $U_{\text {tot }}$. If the built-in operation in Fig. 1 is an image rotation $U=R(\theta)$, the combined matrix $U_{\text {tot }}$ is equal to unity and hardly interesting. If the built-in operation is an image reflection $U=M(\phi)=R(2 \phi) M_{y}$, the combined operation $U_{\text {tot }}$ $=R(4 \phi)$ is a rotation over an angle $4 \phi$. If the interferometer contains more than two mirrors, it can still be reduced to one of these two generic cases by absorbing the extra reflections in the effective image transformation $U$ in Fig. 1 .

We will study the case where the effective image transformation $U$ is an image reflection in more detail. We sometimes call this system an "odd- $R$ " interferometer, to indicate that it operates as an interferometer containing an odd number of mirrors in between crystal and beam splitter and an image rotator $R(\theta)=M(\theta / 2) M_{y}$ in one of its arms. We will evaluate Eq. (7) for this "odd- $R$ " geometry, where the relation $U_{\text {tot }}=R(2 \theta)$ yields $\left|\frac{1}{2}\left(U_{\text {tot }}-1\right) \mathbf{x}_{2}\right|=\sin \theta\left|\mathbf{x}_{2}\right|$. We consider a geometry that comprises a Gaussian TEM $_{00}$ pump beam and a "hard-edged" circular aperture with a top-hat transmission profile $T_{2}\left(\mathbf{x}_{2}\right)=\Theta\left(1-\left|\mathbf{x}_{2}\right| / a\right)$ of radius $a$ positioned in the far field of this beam $\left(L_{0} \gg z_{0}\right)$. For this geometry, the two-photon bunching visibility [Eq. (7)] becomes

$$
V(\theta)=[1-\exp (-\xi)] / \xi
$$

where $\xi=\frac{1}{2}\left(a / w_{z}\right)^{2} \sin ^{2} \theta$. Note that the predicted two-photon visibility $V(\theta)$ is symmetric under inversion of the rotation angle $[V(-\theta)=V(\theta)]$ and periodic in $\pi$ instead of $2 \pi$ radian $[V(\theta+\pi)=V(\theta)]$.

Our key result of Eq. (11) quantifies the effect of "spatial labeling" on the two-photon interference. If the aperture $T_{2}(\mathbf{x})$ is much smaller than the transverse correlation length $w_{z}$ of the pump, we expect $V(\theta) \approx 1$ irrespective of the rotational angle $\theta$, as the diffraction limit of the aperture frustrates the observation of any image rotation or reflection. If the aperture is much larger, diffraction will be less restrictive and $V(\theta)$ will decay rapidly away from $\theta=0$. The two-photon interference should disappear if one can distinguish the signal from the idler path based on any conceivable photo position measurement at the detector side, even if that measure- 
ment is not actually performed but only possible in principle. Mathematically, the criteria for spatial labeling translates into $\sqrt{2 \xi}=\left(a / w_{z}\right) \sin (\theta) \gg 1$.

\section{DISCRETE MODAL ANALYSIS}

\section{A. Schmidt decomposition of the detected two-photon amplitude}

In the previous section we have analyzed two-photon interference in a two-photon interferometer with an image transformation $U$ in one of its arms (see Fig. 1). We considered the case of a rotationally symmetric pump profile and an orthogonal image transformation matrix $U$, comprising any combination of image reflections and rotations as visualized in Fig. 2. We found out that the two-photon bunching visibility is only affected by $U$ if $U$ is an image reflection $M(\theta / 2)$, which is equivalent to an image rotation in combination with an extra mirror $R(\theta) M_{y}$. An expression for $V(\theta)$ is given by Eq. (11) for detection through a 'hard-edged' circular aperture in front of one of the detectors. Our analysis that led to this result was based on calculations of the continuous twophoton amplitude in the quasimonochromatic paraxial thincrystal limit.

In this subsection we will analyze the two-photon interference that leads to Eq. (11) from a different perspective, namely by decomposing the continuous two-photon amplitude into a countable set of discrete spatial modes. We will consider the detected two photon amplitude [15] instead of the generated two-photon amplitude [16,17]. As we will show, the rotational symmetry of the pump and the apertures allows for a decomposition of the detected two-photon amplitude in a Fourier series of orbital angular momenta. This azimuthal decomposition is a first step towards a full Schmidt decomposition $[16,18,19]$ of the detected field. Our Schmidt decomposition of the detected field is mathematically equivalent to the Schmidt decomposition of the generated field as performed in Ref. [16].

We have shown in the previous section that rotational symmetry of the pump field ( $l=0$ pumping) leads to invariancy of the two-photon amplitude under any orthogonal transformation $U$ on both beam coordinates, i.e., that $A_{g}\left(U \delta \mathbf{x}_{s}, U \delta \mathbf{x}_{i}\right)=A_{g}\left(\delta \mathbf{x}_{s}, \delta \mathbf{x}_{i}\right)$. Based on this symmetry we can rewrite $A_{g}\left(\delta \mathbf{x}_{s}, \delta \mathbf{x}_{i}\right)$ as $A_{g}\left(r_{s}, r_{i}, \phi_{s i}\right)$, where $\phi_{s i} \equiv \phi_{s}-\phi_{i}$. Here, we have introduced polar coordinates $\delta \mathbf{x}_{s, i} \leftrightarrow\left(r_{s, i}, \phi_{s, i}\right)$, where $\phi$ is the angle with the $y$ axis and the sign of $\phi$ is defined in analogy with the definition of $R(\theta)$ in Fig. 2. The detected two-photon amplitude is obtained by including the spatial filtering of two rotationally symmetric apertures $T_{s, i}\left(r_{s, i}\right)$ in the signal and idler beam (see Fig. 3). We analyze the angular dependence of this detected field by decomposing it in a Fourier series of orbital angular momenta $l$, via

$$
\begin{aligned}
A_{\text {in }}\left(r_{s}, r_{i}, \phi_{s i}\right) & \equiv \sqrt{T_{s}\left(r_{s}\right) T_{i}\left(r_{i}\right)} A_{g}\left(r_{s}, r_{i}, \phi_{s i}\right) \\
& =A \sum_{l=-\infty}^{\infty} F_{l}\left(r_{s}, r_{i}\right) \sqrt{P_{l}} e^{i l \phi_{s i} / 2 \pi},
\end{aligned}
$$

where $A^{2} \equiv \iint\left|A_{\text {in }}\left(\delta \mathbf{x}_{s}, \delta \mathbf{x}_{i}\right)\right|^{2} d \delta \mathbf{x}_{s} d \delta \mathbf{x}_{i}$ is the "average amplitude squared." Furthermore, we have $F_{l}\left(r_{s}, r_{i}\right)=F_{-l}\left(r_{s}, r_{i}\right)$ and

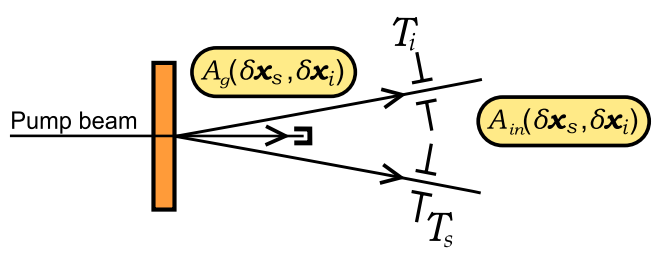

FIG. 3. (Color online) Graphical representation of what we call the detected two-photon amplitude $A_{\text {in }}\left(\delta \mathbf{x}_{s}, \delta \mathbf{x}_{i}\right)$ in relation to the generated two-photon amplitude $A_{g}\left(\delta \mathbf{x}_{s}, \delta \mathbf{x}_{i}\right)$.

$P_{-l}=P_{l}$, because of mirror symmetry. The functions $F_{l}\left(r_{s}, r_{i}\right)$ are normalized via [16]

$$
\int_{0}^{\infty} \int_{0}^{\infty}\left|F_{l}\left(r_{s}, r_{i}\right)\right|^{2} r_{s} r_{i} d r_{s} d r_{i}=1
$$

so that $\Sigma P_{l}=1$.

Equation (12) is a first step towards a full Schmidt decomposition of the detected two-photon amplitude. This decomposition can be completed by expanding [16]

$$
F_{l}\left(r_{s}, r_{i}\right) \sqrt{r_{s} r_{i}}=\sum_{p=0}^{\infty} \sqrt{\gamma_{l, p}} f_{l, p}\left(r_{s}\right) g_{l, p}\left(r_{i}\right)
$$

where the radial mode number $p$ quantifies the number of nodal lines in the radial profile of $f_{l, p}\left(r_{s}\right)$ and $g_{l, p}\left(r_{i}\right)$. The functions $f_{l, p}\left(r_{s}\right)$ and $g_{l, p}\left(r_{i}\right)$ are normalized via the standard inner product so that $\Sigma_{p} \gamma_{l, p}=1$. The full Schmidt decomposition of the detected two-photon amplitude now reads

$$
A_{\text {in }}\left(\delta \mathbf{x}_{s}, \delta \mathbf{x}_{i}\right)=A \sum_{l=-\infty}^{\infty} \sum_{p=0}^{\infty} \sqrt{\lambda_{l, p}} u_{l, p}\left(\delta \mathbf{x}_{s}\right) v_{-l, p}\left(\delta \mathbf{x}_{i}\right),
$$

where $\lambda_{l, p} \equiv P_{l} \gamma_{l, p}$ and $u_{l, p}\left(\delta \mathbf{x}_{s}\right) \equiv e^{i l \phi_{s}} f_{l, p}\left(r_{s}\right) / \sqrt{2 \pi r_{s}}$ and $v_{l, p}\left(\delta \mathbf{x}_{i}\right) \equiv e^{i l \phi_{i}} g_{l, p}\left(r_{i}\right) / \sqrt{2 \pi r_{i}}$.

We now return to the HOM interference setup visualized in Fig. 1 with an image transformation $U=R(\theta) M_{y}$. We reposition the apertures $T_{s}\left(r_{s}\right)$ and $T_{i}\left(r_{i}\right)$ in front of the detectors 2 and 1, respectively. Because the generated field $A_{g}\left(\delta \mathbf{x}_{s}, \delta \mathbf{x}_{i}\right)$ is invariant under a coordinate swap $\delta \mathbf{x}_{s} \leftrightarrow \delta \mathbf{x}_{i}$, we can write the two-photon amplitude behind the apertures $T_{1}$ and $T_{2}$ in terms of the detected two-photon amplitude, i.e.,

$$
\begin{aligned}
A_{\mathrm{HOM}}\left(\mathbf{x}_{1}, \mathbf{x}_{2}\right)= & T_{\mathrm{BS}} e^{-i(1 / 2) \Delta \omega \tau} A_{\mathrm{in}}\left(r_{2}, r_{1}, \phi_{1}+\phi_{2}-\theta\right) \\
& -R_{\mathrm{BS}} e^{i(1 / 2) \Delta \omega \tau} A_{\mathrm{in}}\left(r_{2}, r_{1}, \phi_{1}+\phi_{2}+\theta\right),
\end{aligned}
$$

where $\Delta \omega \equiv \omega_{1}-\omega_{2}$ is the frequency difference between photons 1 and 2 and $\tau \equiv\left(L_{s}-L_{i}\right) / c$ is the time delay difference in the interferometer. The only difference between Eq. (4) and Eq. (16) is that the latter incorporates the transmission profiles of the detection apertures whereas Eq. (4) does not.

The two-photon bunching visibility as defined in Sec. II is now easily calculated by using the azimuthal Schmidt decomposition of the detected two-photon amplitude as given in Eq. (12). By substituting Eq. (12) in Eq. (16), assuming a balanced beam splitter $R_{\mathrm{BS}}=T_{\mathrm{BS}}=\frac{1}{2}$, and using the prescriptions of Eqs. (5) and (6) one quickly finds 


$$
V(\theta)=\sum_{l=-\infty}^{\infty} P_{l} \cos (2 l \theta) .
$$

In other, words, a measurement of $V(\theta)$ with the HOM setup as visualized in Fig. 1 [with $U=R(\theta) M_{y}$ ] reveals the azimuthal Schmidt coefficients $P_{l}$ of the detected two-photon amplitude. This key result will be discussed in more detail in Sec. III C.

The OAM weights $P_{l}$ depend on the size and radial shape of the circular detection apertures $T_{1,2}$ in relation to the profile of the pump laser in the detection plane, as these determine the detected two-photon amplitude $A_{\mathrm{in}}\left(r_{s}, r_{i}, \phi_{s i}\right)$ and its angular Fourier components $A F_{l}\left(r_{s}, r_{i}\right) \sqrt{P_{l}} / 2 \pi$. These OAM weights are often difficult to calculate. For our geometry with a Gaussian pump and a single hard-edged aperture, we did not find analytic expressions for $P_{l}$, as we could not solve the Fourier decomposition of Eq. (11) or Eq. (12) analytically.

\section{B. Modal decomposition and the Schmidt number}

In this subsection, we will introduce a convenient coordinate free bra-ket notation for the detected two-photon state (see Fig. 3) and use the Schmidt decomposition to quickly rederive the previous results of Eq. (16) and Eq. (17). We will also introduce two different Schmidt numbers.

A Schmidt decomposition of the detected two photon state $|\Psi\rangle_{\text {in }}$ in bra-ket form is

$$
|\Psi\rangle_{\text {in }}=\sum_{\mu} \sqrt{\lambda_{\mu}}\left|u_{\mu}\right\rangle \otimes\left|v_{\mu}\right\rangle,
$$

where $\left\{\left|u_{\mu}\right\rangle\right\}$ and $\left\{\left|v_{\mu}\right\rangle\right\}$ are two sets of orthogonal mode functions, which are identical only if the aperture profiles $T_{s}$ and $T_{i}$ are identical. The effective number of modes involved in this decomposition is defined by the so-called (2D) Schmidt number

$$
K_{2 \mathrm{D}} \equiv \frac{\left(\sum_{\mu} \lambda_{\mu}\right)^{2}}{\sum_{\mu} \lambda_{\mu}^{2}} .
$$

The rotation symmetry of the detected two-photon amplitude $A_{\text {in }}\left(U \delta \mathbf{x}_{s}, U \delta \mathbf{x}_{i}\right)=A_{\text {in }}\left(\delta \mathbf{x}_{s}, \delta \mathbf{x}_{i}\right)$ allows one to separate the mode index $\mu$ into an azimuthal mode number $l$ and a radial mode number $p$. It also enforces the conservation of OAM in the paraxial SPDC process [20] and changes the modal decomposition of Eq. (18) to

$$
\left.|\Psi\rangle_{\text {in }}=\sum_{l=-\infty}^{\infty} \sum_{p=0}^{\infty} \sqrt{\lambda_{l, p}} l, p\right\rangle^{\prime} \otimes|-l, p\rangle^{\prime \prime},
$$

where $|l, p\rangle^{\prime}$ and $|-l, p\rangle^{\prime \prime}$ are the LG-like Schmidt eigenmodes of the detected two-photon amplitude. This equation is the bra-ket notation of Eq. (15), where $|l, p\rangle^{\prime}$ and $|-l, p\rangle^{\prime \prime}$ correspond to the functions $u_{l, p}\left(\delta \mathbf{x}_{s}\right)$ and $v_{-l, p}\left(\delta \mathbf{x}_{i}\right)$, respectively. As the amplitude coefficients $\sqrt{\lambda_{l, p}}$ already contain the effects of aperture filtering, they will decrease rapidly both for high $p$ and high $l$ values (high $l$-states are quite extended even for $p=0)$. We define the OAM probability as $P_{l}$ $\equiv \Sigma_{p} \lambda_{l, p}$ and the related azimuthal Schmidt number as

$$
K_{\mathrm{az}} \equiv \frac{1}{\sum_{l} P_{l}^{2}},
$$

for $\Sigma_{l} P_{l}=1[21,26]$. The relation between the azimuthal Schmidt number $K_{\mathrm{az}}$ and the full 2D Schmidt number $K_{2 \mathrm{D}}$ depends (somewhat) on the shape of the detection aperture.

We now return to the HOM interference setup visualized in Fig. 1 with an image transformation $U$. Starting from the modal decomposition of Eq. (20), it is relatively easy to apply the rotation and mirror operations that are needed to evaluate the doubly-reflected and doubly-transmitted field and the visibility of their interference. For the "even $R$ " geometry $[U=R(\theta)]$, the generated $(l,-l)$ pairs are also detected as $(l,-l)$ pairs behind the beam splitter and we expect good two-photon interference, i.e., $V(\theta=1)$, at any rotation angle. For the "odd $R$ " geometry $\left[U=R(\theta) M_{y}\right]$, the OAM inversion produced by the extra mirror leads to the detection of $(l, l)$ and $(-l,-l)$ pairs instead. As the OAM at the rotator is now different for the doubly-reflected and doublytransmitted path, so is the effect of rotation. For rotation over an angle $\theta$ the combined two-photon state after HOM interference can now be written as

$$
\begin{aligned}
|\Psi\rangle_{\mathrm{HOM}}= & \sum_{l, p}\left[T_{\mathrm{bs}} \sqrt{\lambda_{l, p}} e^{-i[l \theta+(1 / 2) \Delta \omega \tau]}-R_{\mathrm{bs}} \sqrt{\lambda_{-l, p}} e^{i[l \theta+(1 / 2) \Delta \omega \tau]}\right] \\
& \times|l, p\rangle^{\prime} \otimes|l, p\rangle^{\prime \prime} .
\end{aligned}
$$

This is the bra-ket notation of Eq. (16). Next, we assume a balanced beam splitter $\left(R_{\mathrm{bs}}=T_{\mathrm{bs}}=\frac{1}{2}\right)$ and use the reflection symmetry $\lambda_{l, p}=\lambda_{-l, p}\left(\right.$ and $\left.P_{l}=P_{-l}\right)$ to obtain

$$
V(\theta)=\sum_{l=-\infty}^{\infty} P_{l} \cos (2 l \theta)
$$

where we normalized to $\Sigma_{l} P_{l}=1$ [equivalent to $V(0)=1$ ]. With the convenient bra-ket notation, we thus recover the important Eq. (17) in only a few steps.

\section{Physical significance of $V(\theta)$}

Equation (23) shows how the observed visibility $V(\theta)$ is a weighted sum over contributions from groups of $l$ modes, each contribution oscillating between $V_{l}=1$ (HOM dip) and $V_{l}=-1$ (HOM peak) with its own angular dependence $\cos (2 l \theta)$. It thereby shows how the visibility $V(\theta)$ and the modal distribution $\left\{P_{l}\right\}$ are related via a simple Fourier series. As a Fourier transformation of $V(\theta)$ directly yields the full OAM distribution $\left\{P_{l}\right\}$, it thus provides for a complete characterization of the angular structure of the two-photon amplitude.

The azimuthal Schmidt number $K_{\mathrm{az}}$ is a measure for the angular structure in the detected two-photon amplitude. More precisely, by averaging $V(\theta)^{2}$ over the full rotation range one finds 

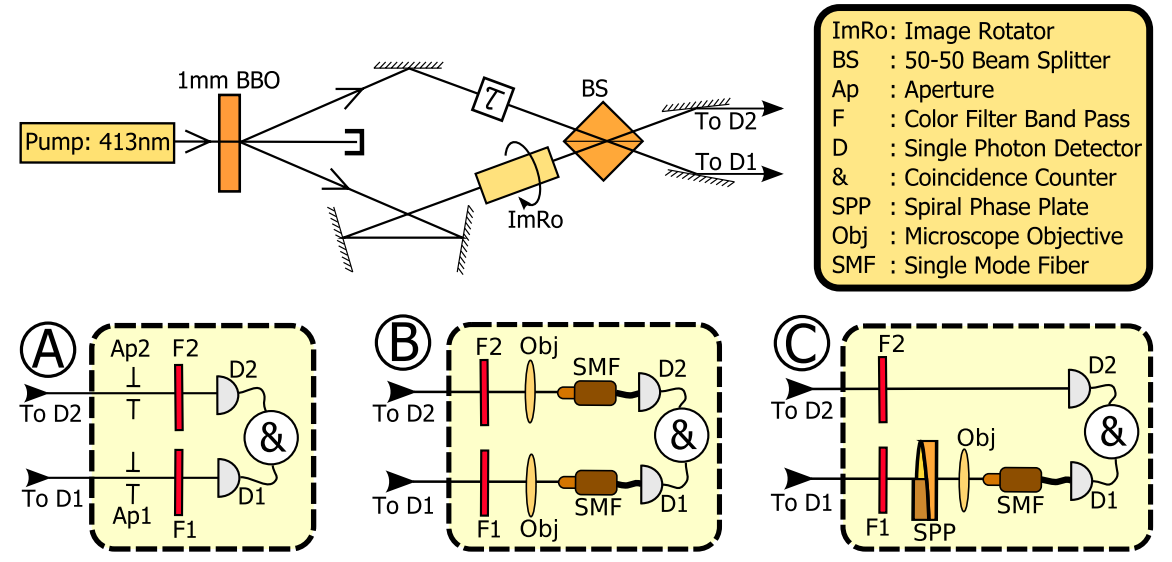

When $V(\theta)$ remains close to $V(0)=1$ over its full range, this relation gives $K_{\mathrm{az}} \approx 1$. When $V(\theta)=\cos (2 l \theta)$ this relation gives $K_{\mathrm{az}}=2$ as expected for $P_{l}=P_{-l}=\frac{1}{2}$. When $V(\theta) \approx 1$ only in a very limited range around $\theta=0$ and zero for all other angles, $K_{\mathrm{az}} \gg 1$ is inversely proportional to the angular width of $V(\theta)$.

In one of our experiments we use a single-mode detector that only selects photons with a specific OAM value $l_{d}$. We predict that the coincidence rate versus time delay $R_{\mathrm{cc}}(\theta, \tau)$ now contains both a symmetric and (surprisingly) also an antisymmetric part with respect to time delay $\tau$. Using the detected two-photon state after HOM interference of Eq. (22) for a single $l=l_{d}$ number we find

$$
R_{\mathrm{cc}}(\theta, \tau) \propto \int\left[1+\cos \left(\Delta \omega \tau+2 l_{d} \theta\right)\right] T_{\mathrm{tot}}(\Delta \omega) d \Delta \omega .
$$

This equation shows that $R_{\mathrm{cc}}(\theta, \tau)$ will have an antisymmetric component only if $T_{\text {tot }}(\Delta \omega)$ is asymmetric and if $\sin (2 l \theta) \neq 0$. Note that $T_{\text {tot }}(\Delta \omega)$, as defined below Eq. (5), is asymmetric only if the filter transmission spectra $T_{f 1}(\omega)$ and $T_{f 2}(\omega)$ are different. For the two-photon bunching visibility at zero delay, which solely depends on the symmetric part, we find the earlier result of Eq. (23), which now reduces to $V(\theta)=\cos \left(2 l_{d} \theta\right)$.

Finally, one might wonder what the observed visibility $V(\theta)$ tells us about the nature of the spatial entanglement, i.e., whether it proves that the two-photon amplitude is indeed described by the pure state of Eq. (20) with its perfect OAM entanglement. This question is best answered by arguing backwards from hypothetical detected pairs $\left(l_{1}, l_{2}\right)$. For an "even $R$ " interferometer, our experimental observation that $V(\theta) \approx 1$ irrespective of rotation angle indeed proves the conservation of OAM; it shows that the two-photon field at the detectors contains only $(l,-l)$ pairs, as any other pairs $\left(l_{1}, l_{2}\right)$ would introduce an angle dependence of the form $\cos \left(2\left(l_{1}+l_{2}\right) \theta\right)$ in $V(\theta)$. However, as the same result $V(\theta)$ $\approx 1$ would have been obtained for any classical mixture of $(l,-l)$ pairs, this observation does not prove the existence of quantum entanglement. For the "odd- $R$ " interferometer, the observations on $V(\theta)$ discussed in this paper do prove some form of quantum entanglement. It shows that the two-photon amplitude contains only coherent superpositions of the form $|\Psi\rangle_{\text {in }}=|l,-l\rangle+|-l, l\rangle$. Again, we cannot exclude any incoherent mixture of these superposition states.

\section{EXPERIMENTAL RESULTS}

\section{A. Experimental setup}

Our experimental setup, as shown in Fig. 4, is a twophoton (Hong-Ou-Mandel type) interferometer with an odd number of mirrors and an image rotator in one of the arms. A cw krypton-ion laser (Coherent Innova 300) emits $210 \mathrm{~mW}$ at $413.1 \mathrm{~nm}$ in a vertically polarized pure $\mathrm{TEM}_{00}$ mode. The beam is mildly focused $\left(w_{p}=270 \mu \mathrm{m}\right.$ is the radius at $e^{-2}$ of maximum irradiance) on a $1 \mathrm{~mm}$ thick $\beta$ - $\mathrm{BaB}_{2} \mathrm{O}_{4}$ crystal (BBO) with a cutting angle of $29.2^{\circ}$. The crystal is tilted such that we obtain type I spontaneous parametric down conversion (SPDC) where the SPDC-light is emitted in a cone extending over a full opening angle of $2 \theta_{0}=3.2^{\circ}$ around the pump beam. After multiple reflections and a single transit through the image rotator, two opposite parts of this cone are combined on a beam splitter. The crucial angular alignment of this beam splitter is performed with computer-controlled actuators. Behind the 50/50 beam splitter, three different detection geometries can be chosen (described in more detail in the next paragraph). Color filters are positioned in front of the detectors in all detection geometries. The filters are custom-made bandpass filters (Chroma Technology Corporation), filtering around $826 \mathrm{~nm}$ with a FWHM of $5 \mathrm{~nm}$. The detectors in all three detection geometries are single photon sensitive avalanche photodiodes (Perkin Elmer SPCM-AQR14).

Detection geometry $A$ consists of a relatively large (bucket) detector behind an aperture (at $0.10 \mathrm{~m}$ from the beam splitter) in each detection arm. Each detector will collect all the light that passes through its aperture. In detection geometry $B$, each detector is connected to a single-mode fiber collecting only the fundamental Gaussian mode. In detection geometry $C$, one of the detectors is connected to a single-mode fiber in combination with a spiral phase plate (SPP) making it effectively a single-mode $l=1$ detector, 


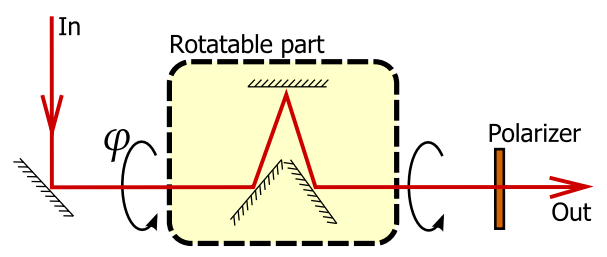

FIG. 5. (Color online) Scheme of the different components of our image rotator, drawn in the $x z$ plane (see Fig. 1). Light enters with an in-plane linear polarization. The rotatable part (drawn here in the $\phi=0$ situation) can be rotated as a whole around the indicated axis, and it is responsible for an image reflection $M(\phi)$ as defined in Fig. 2. The polarizer selects the in-plane polarization.

while the other detecting arm contains a bucket detector. A more detailed description of the $l=1$ single-mode detector and the fabrication of the SPP can be found in Sec. IV B. The apertures (in geometry $A$ ) and the position of the objectives (in geometries $B$ and $C$ ) define the signal and idler path. The beam splitter is positioned on the crossing of the signal and idler path and is angle tuned such that the detection apertures obey mirror symmetry with respect to the beam splitter plane. The signal and idler arm of the interferometer each have a length of $1.6 \mathrm{~m}$. The direction of the pump beam is centered in between the directions of the signal and idler path. The idler arm contains a delay line with which the length of the arm, and hence the relative arrival times of the photons at the beam splitter, can be adjusted.

The microscope objectives in detection geometry $B$ are Leica $10 \times / 0.25$ infinity-corrected objectives with an effective focal length of $20 \mathrm{~mm}$. They are positioned at $0.6 \mathrm{~m}$ from the beam splitter and image the tip of the single-mode fiber (Thorlabs SM800-5.6-125) onto the BBO crystal. The radius (at $e^{-2}$ of maximum irradiance) of this image of the detected mode on the BBO crystal is measured to be $280 \mu \mathrm{m}$. We use slightly different optics in detection geometry $C$, in order to obtain a narrower waist of the detected mode on the BBO crystal. The radius (at $e^{-2}$ of maximum irradiance) of the detected $l=0$ mode, i.e., whenever the SPP is temporarily removed from the apparatus, on the $\mathrm{BBO}$ crystal is now $220 \mu \mathrm{m}$. The radius of the characteristic ring of the detected $l=1$ mode, i.e., whenever the SPP is in the apparatus, is $180 \mu \mathrm{m}$. This value is somewhat larger than the value of $\frac{1}{2} \sqrt{2} \times 220 \mu \mathrm{m}=156 \mu \mathrm{m}$ expected for the $(l=1, p$ $=0)$-mode due to the presence of higher order $p$-modes.

The image rotator includes five optical components as shown in Fig. 5. The rotatable part is responsible for an image reflection $M(\phi)$ in a line making an angle $\phi$ with the $y$ axis (see Fig. 2). It consists of three discrete mirrors instead of a commercially available glass Dove prism, in order to avoid any detrimental effects of wavelength dispersion. We are able to align the rotatable part within $\pm 0.2 \mathrm{mrad}$ in the far field and $\pm 0.5 \mathrm{~mm}$ in the near field, measured over a full rotation. The fixed mirror on the left causes an image reflection $M(0)$ so that the combined action of the unit is a rotation $R(\theta)=R(2 \phi)=M(\phi) M(0)$.

Although an image rotation is generally accompanied by a polarization rotation [22], our rotator transfers at most only $8 \%$ of the power into the orthogonal polarization. This con- venient property is obtained by using silver mirrors (protected by a thin $\mathrm{SiO}_{2}$ cover layer) instead of dielectric mirrors. The measured phase difference between the (threefold) reflected $s$ - and $p$-polarized light is $\phi_{s-p}=0.81 \pi$ [23], which is sufficiently close to the ideal value of $\phi_{s-p}=\pi$ needed for a polarization-insensitive rotator. As both polarization components have the same spatial profile, we simply remove this small unwanted orthogonal component with a fixed polarizer (see Fig. 5).

\section{B. Spiral phase plate}

A spiral phase plate (SPP) is a transparent plate whose thickness increases proportional to the azimuthal angle [24]. It imposes an azimuth-dependent optical retardation on the optical field. Our SPP is custom made by Philips Research Laboratories with dimensions suited for our application; the imposed optical retardation over a full rotation equals one optical cycle of the $826.2 \mathrm{~nm}$ SPDC-light. The SPP operates as a lowering operator on $l$-numbers of the incoming modes at this wavelength. In detection geometry $C$, a single-mode fiber is used to detect solely the fundamental Gaussian mode $(l=0)$ behind the SPP which implies that it solely detects an $l=1$ mode in front of the SPP.

The SPP is manufactured using photo replication technology [25]. To this end a high accuracy brass mold, the negative of the SPP we wish to produce, is machined using a programmable computer driven diamond tool. A transparent copy of the mold is obtained by using a reactive monomer encapsulated between the mold and a glass cover plate. The final SPP is obtained after polymerization of the monomer by UV radiation. The demand for an optical retardation of $826 \mathrm{~nm}$ in one cycle requires proper definition of step height and refractive index. We use a step height of $1.66 \mu \mathrm{m}$ and a refractive index of 1.50 . Some technical details on the production process are as follow: We use an adhesion promoter $\gamma$-(methacryloyloxypropyl)trimethoxysilane to allow for a firm coupling between the resulting polymer and the cover. We use a mixture of poly(ethyleneglycol) dimethacrylate with a refractive index of 1.48 and Ebecryl 604 (75\% epoxyacrylate in hexanedioldiacrylate, a product of UCB chemicals) with a refractive index of 1.54 in a ratio of 2:1 to obtain an effective index of 1.50 . To enable the photopolymerization reaction $2 \%$ of a mixture of photoinitiators (Irgacure 651 and Irgacure 184) was added.

\section{Alignment}

We will discuss the alignment procedure in more detail because we think it can be helpful for anyone who wants to reproduce the experiment. We consider the position of the beam splitter as well as the position of the pump spot on the $\mathrm{BBO}$ crystal as fixed. Leaving out the rotator from the discussion we need to deal with the following degrees of freedom: angle of the pump beam $\theta_{p}$ and the angle of the beam splitter $\theta_{\mathrm{BS}}$. In detection geometry $A$ we additionally need to consider the position of the apertures. In detection geometries $B$ and $C$ we additionally need to consider the positions of the objectives as well as the positions of the fibers (determining the position and angle of the detected mode on the 
crystal), assuming that the SPP is positioned correctly in geometry $C$. The correct alignment of $\theta_{p}$ and $\theta_{\mathrm{BS}}$ are independent of the choice of detection geometry. Therefore, we are allowed to switch to another detection geometry halfway the alignment procedure.

We start the alignment procedure by aligning the angle of the beam splitter in detection geometry $B$. We do this by first moving the fiber in the transverse plane in order to maximize the single count rate of each fiber for the corresponding transmission channel only. Secondly, we tune $\theta_{\mathrm{BS}}$ to maximize the single count rates of the reflection channels. The accuracy of this alignment step is $\Delta \theta_{\mathrm{BS}}= \pm 70 \mu \mathrm{rad}$. The correct alignment of the angle of the beam splitter depends on the position of the pump spot on the BBO as well as the alignment of the optics in the interferometer arms. From now on we do not change either of these.

The next goal is to align the angle of the pump beam. We control $\theta_{p}$ with a mirror that is positioned very close to the $\mathrm{BBO}$ crystal. Changing the angle of the pump beam with this mirror has a negligible effect on the position of the pump beam on the BBO crystal. The angle of the pump beam must become centered in between the detected directions in the signal and idler arm. We do this by maximizing the coincidence count rate $R_{\mathrm{cc}, \text { ref }}$ far away from HOM interference. Note that one may only perform this operation if the apertures or objectives are positioned correctly, obeying mirror symmetry with respect to the beam splitter plane. This positioning of apertures (detection geometry $A$ ) or fiber-detectors detectors (detection geometry $B$ and $C$ ) is done by eye, using a visible HeNe laser.

To measure the correct visibility in detection geometry $A$ with a large aperture diameter, an even higher precision of the alignment of the angle of the beam splitter is required $\left(\Delta \theta_{\mathrm{BS}}= \pm 40 \mu \mathrm{rad}\right.$ set by the diffraction limit). We improve the alignment by simply minimizing the coincidence count rate inside the HOM dip in detection geometry $A$. One may only apply this alignment technique if all the other components are aligned correctly, for minimizing the coincidence count rate can sometimes also be achieved by misaligning any other component in the setup.

The alignment of the fiber-detector in detection geometry $C$ deserves some extra attention. The problem is that placing the spiral phase plate (SPP) in front of the single-mode fiber will slightly shift the central position of the detected mode on the BBO crystal $(\sim 1 \mathrm{~mm})$ due to a small wedge in the SPP. As a consequence, one has to reposition the fiber with respect to the microscope objective in order to get the position of the detected mode centered at the pump beam again. From experimental experience we know that the shape of $R_{\mathrm{cc}}(\tau)$, and hence also the visibility, is extremely sensitive to the position of the projection of the detected $l=1$ mode on the BBO crystal. A transverse shift of only $25 \mu \mathrm{m}$ on the BBO can lead to a decrease of the HOM visibility of $9 \%$ points in the case of an image rotation of $\theta=90^{\circ}$. This means that an alignment precision below $\Delta \theta_{\operatorname{det}}<10 \mu \mathrm{rad}$ is required. We achieve this accuracy by imaging the projection of the detected $l=1$ mode on the BBO crystal onto a CCD camera.

We want to stress that fine tuning the position fiber in detection geometry $C$ by optimizing two-photon interference

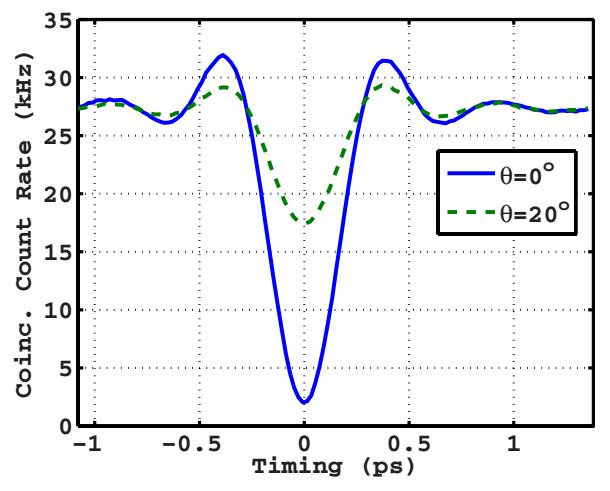

FIG. 6. (Color online) Two-photon coincidence count rate versus the time delay $\tau$ for two different rotation angles. The measurements are performed in detection geometry $A$ (see Fig. 4) where one aperture has a diameter of $10 \mathrm{~mm}$ and the other aperture is open. The two-photon bunching visibilities are $92 \%$ and $36 \%$ for $\theta=0^{\circ}$ and $\theta=20^{\circ}$, respectively.

effects (i.e., maximizing the absolute visibility) may result in an incorrectly aligned system. Figure 10 shows two results which are obtained with an incorrectly positioned projection of the detected $l=1$ mode on the BBO crystal. The figure makes clear that an incorrectly aligned system may give an absolute visibility that is even higher than the visibility that would have been obtained with a correctly aligned system.

\section{Experimental results for detection through circular apertures}

We have measured the two-photon bunching visibility as a function of image rotation with the setup shown in Fig. 4. In this subsection we present the results obtained in detection geometry $A$ (detection through circular apertures) and in detection geometry $B$ (detection of solely the fundamental Gaussian mode). We compare these measurements with the theoretical predictions and we determine the azimuthal Schmidt number $K_{\mathrm{az}}$, which is the effective dimensionality of the entanglement in orbital angular momentum.

The effect of an image rotation on the two-photon bunching visibility is clearly illustrated in Fig. 6. The figure shows measurements of the coincidence count rate as a function time delay for two different rotation angles $\theta=0^{\circ}$ and $\theta$ $=20^{\circ}$ in detection geometry $A$ (aperture diameter of $10 \mathrm{~mm}$ ). Both cases exhibit a drop of the coincidence count rate near $\tau=0$ reaching almost ideal photon bunching $(V=92 \%)$ in the nonrotated case. The dip in the case of $\theta=20^{\circ}$ on the other hand, has a strongly reduced visibility of only $36 \%$.

The two visibilities extracted from Fig. 6 correspond to two points in Fig. 7. In this figure we have plotted the bunching visibility $V(\theta)$ as a function of the rotation angle for various aperture diameters (detection geometry $A$ ) and for two fiber-coupled detectors (detection geometry $B$ ). As expected, we observe almost ideal photon bunching for all detection geometries as long as we apply no image rotation. If we apply a certain image rotation, however, the two-photon bunching visibility becomes different for different detection geometries. The measurement with fiber-coupled detectors 


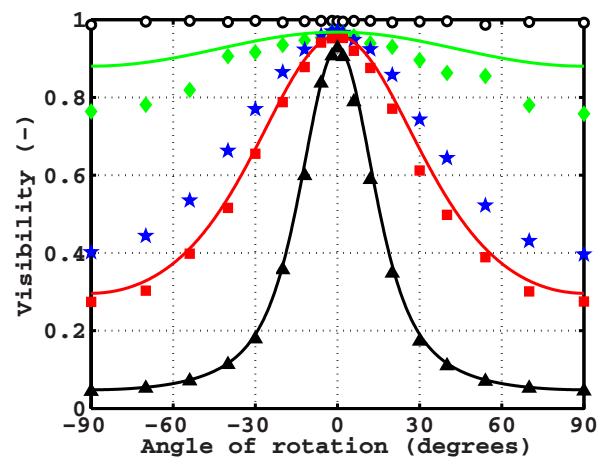

FIG. 7. (Color online) Measured two-photon bunching visibility versus angle of rotation for various detection geometries (see Fig. 4): Two single-mode fibers (circles), 1-mm-open apertures (diamonds), 4-mm-4-mm apertures (stars), 4-mm-open apertures (squares) and 10-mm-open apertures (triangles). The three solid curves are predicted by theory [Eq. (11)] and correspond from top till bottom to the measurements of the diamonds, the squares, and the triangles, respectively.

serves as a reference measurement, demonstrating that the bunching visibility of the rotational symmetric fundamental Gaussian mode is independent of the angle of rotation (remaining $V>98.7 \%$ ). Our measurements in detection geometry $A$ show that a larger aperture corresponds to a narrower peak of $V(\theta)$ around $\theta=0$. This is in agreement with our expectations based on the calculation of the continuous twophoton amplitude [Eq. (11)]. The figure shows theoretical curves of $V(\theta)$ for aperture diameters of $1 \mathrm{~mm}, 4 \mathrm{~mm}$, and $10 \mathrm{~mm}$. The latter two are in good agreement with the measurements, but the $1 \mathrm{~mm}$ case shows a slight deviation which we believe is the result of imperfect alignment.

Apart from the three cases mentioned above, where the second aperture is set completely open, we have also measured $V(\theta)$ in the case where both apertures are set to a diameter of $4 \mathrm{~mm}$ (see Fig. 7). Closing the second aperture to the size of the first one slightly broadens the $V(\theta)$ curve. The detection of one photon within an aperture minimizes the position uncertainty of the other (brother) photon to twice the width of the pump beam in the detection plane [see Eq. (3)]. The fact that the position of the second photon is still not completely fixed after detection of the first one explains why it matters whether or not an identical aperture in front of the second detector is present. Closing the second aperture to the same size indeed also reduces the reference coincidence rate $R_{\mathrm{cc}}(\infty)$. We have not plotted the corresponding theoretical curve in Fig. 7 simply because we have no analytic expression for $V(\theta)$ in a detection geometry with two equally closed apertures.

Now what do the measurements in Fig. 7 tell us about the generated two-photon state? From symmetry arguments (Gaussian pump beam, paraxial angle of the SPDC cone, and circular detection apertures) we know that we can write the detected part of the generated two-photon state in the form of Eq. (20). By applying a cosine transform on the measured curve $V(\theta)$ we find all the orbital angular momentum (OAM) probability coefficients $P_{l}$ conform Eq. (23). This means that a measurement of $V(\theta)$ provides a complete characterization

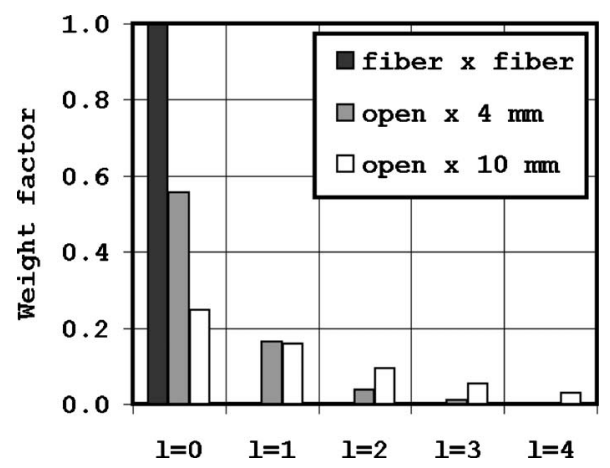

FIG. 8. Measured weight factors $P_{l}$ of the "orbital angular momentum" terms in the entangled two-photon state for the three specified detection geometries. Not shown are the weight factors for $l>4$ and negative $l$, for which $P_{-l}=P_{l}$.

of the high dimensional entanglement that exists between the orbital angular momenta of the two photons.

We have performed such modal decompositions on our measured curves $V(\theta)$. The resulting coefficients $P_{l}$ for three (of five) detection geometries are shown in Fig. 8. It is clearly visible that the contributions of the smallest $l$-numbers become more dominant if the detection apertures become narrower. The measurement with single mode fibers (detection geometry $B$ ) serves as a reference, and shows that the detected two-photon state contains only photons with zero orbital angular momentum, i.e., with $l=0$. From the measured values of $P_{l}$ we calculate the azimuthal Schmidt number [using Eq. (24)] which is the effective number of modes that participate in the entanglement. The values are listed in Table I. For detection through single mode fibers (detection geometry $B$ ) we find $K_{\mathrm{az}}=1.01 \pm 0.01$ and in detection geometry $A$ the azimuthal Schmidt number ranges between $1.26 \pm 0.06$ and $7.3 \pm 0.3$ depending on the aperture diameter.

\section{E. Experimental results for $l=1$ detection}

In this subsection we present the measurements that we have performed in detection geometry $C$, where one of the detectors is coupled to a single-mode $l=1$ selector (see Fig. 4). Again, we have measured the coincidence count rate $R_{\text {cc }}(\tau)$ as a function of delay time. The results for various

TABLE I. Measured azimuthal Schmidt number $\left(K_{\mathrm{az}}\right)$ of the detected two-photon state for various detection geometries (see Fig. 4 ). The first row is measured in detection geometry $B$; the other four rows are measured in detection geometry $A$, for which the aperture diameters are specified.

\begin{tabular}{ccc}
\hline \hline Detector 1 & Detector 2 & $K_{\mathrm{az}}$ \\
\hline SM-fiber & SM-fiber & $1.01 \pm 0.01$ \\
$1 \mathrm{~mm}$ & open & $1.26 \pm 0.06$ \\
$4 \mathrm{~mm}$ & $4 \mathrm{~mm}$ & $2.08 \pm 0.08$ \\
$4 \mathrm{~mm}$ & open & $2.7 \pm 0.1$ \\
$10 \mathrm{~mm}$ & open & $7.3 \pm 0.3$ \\
\hline \hline
\end{tabular}




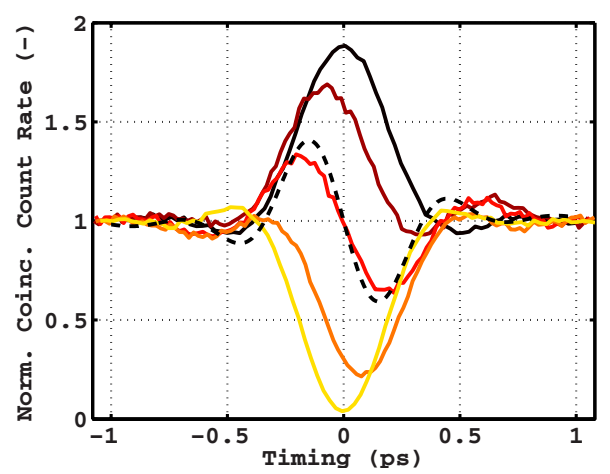

FIG. 9. (Color online) Coincidence count rate versus time delay $\tau$, measured in a single-mode $(l=1)$ detection geometry, for various angles of rotation $\theta$. The five solid curves (from top to bottom) are measured at $\theta=90^{\circ}, 67.5^{\circ}, 45^{\circ}, 22.5^{\circ}$, and $0^{\circ}$, respectively. The dotted curve is calculated for $\theta=45^{\circ}$, making use of Eq. (25) and the measured spectral transmissions of the bandpass filters.

angles of rotation are presented in Fig. 9. As expected from Eq. (23) we observe photon bunching in the case of no image rotation and photon anti-bunching if a rotation of $\theta=90^{\circ}$ is applied.

For angles between $0<\theta<90^{\circ}$ we observe an asymmetric curve for $R_{\mathrm{cc}}(\tau)$. This phenomenon can be fully explained from the spectral shape of the color filters. The theoretical shape of $R_{\mathrm{cc}}(\theta, \tau)$ is given by Eq. (25) from which we conclude that $R_{\mathrm{cc}}(\tau)$ will have an antisymmetric component only if the filters are not exactly identical, making $T_{\text {tot }}(\omega)$ asymmetric, and if $\sin (2 \theta) \neq 0$. We had intended to use identical filters; however, due to the limited fabrication accuracy, the transmission spectra of the two filters were slightly different. One of the filters has a transmission spectrum that is centered around $\lambda=824.7 \mathrm{~nm}$ instead of $\lambda=826.2 \mathrm{~nm}$, whereas the other filter is neatly centered around $\lambda=826.2 \mathrm{~nm}$. This difference is responsible for the antisymmetric component in $R_{\mathrm{cc}}(\tau)$ in Fig. 9.

The dotted curve shows the calculated coincidence count rate for $\theta=45^{\circ}$ as expected from Eq. (25) using measured spectral transmission functions of the filters. The strength of the contribution of the asymmetric component in $R_{\mathrm{cc}}(\tau)$ is in excellent agreement with our measurements. Serving as an extra check of Eq. (25), we have also exchanged the two filters and observe, as expected, an asymmetry of $R_{\mathrm{cc}}(\tau)$ that is flipped around the vertical $\tau=0$ axis.

From the measured curves of $R_{\mathrm{cc}}(\tau)$ for various angles of rotations, we have extracted the two-photon bunching visibility defined as $V(\theta) \equiv 1-R_{\mathrm{cc}}(\tau=0) / R_{\mathrm{cc}}(\tau=\infty)$. According to this definition, $V(\theta)$ depends only on the symmetric component of $R_{\mathrm{cc}}(\tau)$ and hence Eq. (23) is fully applicable to the outcome of our measurement of $V(\theta)$. The resulting values are presented in Fig. 10 and are very well described by the predicted $V(\theta)=\cos (2 \theta)$.

As shown in Fig. 10, we have performed several measurements of $V(\theta)$ twice and observed a small spread which, we believe, is caused by the limited achievable accuracy of the alignment. This limited accuracy of the alignment is the dominant contribution to the uncertainty of \pm 0.05 in the mea-

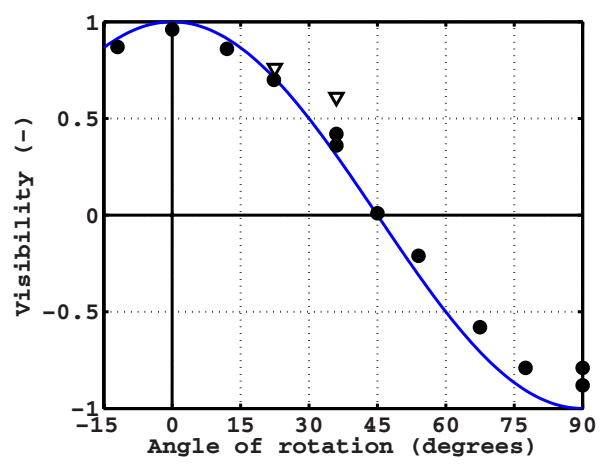

FIG. 10. (Color online) Two-photon bunching visibility versus angle of rotation (circles), where only a single-mode with orbital angular momentum $l=1$ is detected. The triangles are two results obtained with an experimental apparatus that is improperly aligned (see Sec. IV C). The uncertainty of the measured visibility is \pm 0.05 . The solid curve is predicted by theory [Eq. (23)].

surements of $V(\theta)$. We furthermore observe that the twophoton bunching visibility at $\theta=90^{\circ}$ is only $-82 \pm 5 \%$ instead of the predicted $-100 \%$. This may be explained by the observation that our detected $l=1$ mode (detection geometry $C$ ) is not perfectly rotationally symmetric for yet unknown reasons.

\section{CONCLUDING DISCUSSION}

In conclusion, we have presented and demonstrated a method to obtain a complete characterization of the highdimensional entanglement in orbital angular momentum (OAM) of down-converted photon pairs. The method is based on measurements of the interference in a two-photon interferometer (Hong-Ou-Mandel-type) with an odd number of mirrors and a built-in image rotator. We have theoretically analyzed this method for the case of a rotationally symmetric (Gaussian) pump profile and coincidence detection behind circular apertures, using two different approaches. In the first approach, we derive analytic expressions for the two-photon (bunching) visibility $V(\theta)$ as a function of the rotation angle $\theta$, by spatial integration of the interference in the continuous two-photon amplitude. In the second approach, we use a modal (Schmidt) decomposition of the detected two-photon amplitude to derive a natural Fourier relation between $V(\theta)$ and the weight factors $P_{l}$ of the OAM terms in the entangled two-photon state: High OAM states result in fast changes of $V(\theta)$ with angle, whereas low OAM states produce slower changes. These weight factors are then combined to an azimuthal Schmidt number $K_{\mathrm{az}}$, which quantifies the effective dimensionality of the OAM entanglement. Both approaches can easily be generalized to pump profiles with an orbital angular momentum different from $l=0$.

We have shown that the experimental results are in close agreement with the theoretical predictions. More specifically, an increase in the size of the detection aperture results in an increase in the effective dimensionality $K_{\mathrm{az}}$, which is visible as a reduction in the angular width of $V(\theta)$ around $V(0) \approx 1$. We have tuned the effective dimensionality between $K_{\mathrm{az}}$ 
$=1.01 \pm 0.01$ and $K_{\mathrm{az}}=7.3 \pm 0.3$. Using a single-mode OAMselective $(l=1)$ detector, we have isolated one term in the Fourier expansion and demonstrated a controllable change from photon bunching to photon antibunching. At intermediate settings we have observed highly asymmetric HOM dips $V(\tau)$ versus time delay $\tau$. We attribute this asymmetry to a difference between the two spectral filters in front of the detectors.

The Klyshko picture provides for a simple intuitive way to understand the working principle of our OAM analysis method. The Klyshko approach is based on a time reversal of the path of one of the photons. The full path now runs (backwards in time) from one detector back to the generating crystal and after reflection (forward in time) to the second detector. In the Klyshko picture, the two-photon visibility results from the interference between the clockwise and counterclockwise paths, which are associated with the "doubly reflected" and "doubly transmitted" paths at the beam splitter. Only if the total number of mirrors in the interferometer is odd, will these two paths acquire an opposite image rotation, which in turn leads to a reduction of the two-photon visibility. For an even number of mirrors the image rotation is identical for both paths.

One might wonder why our experimental results are limited to $K_{\mathrm{az}} \leqslant 7.3 \pm 0.3$. The prime reason is the limited size of our pump spot. The simplest way to increase the Schmidt numbers $K_{\mathrm{az}}$ and $K_{2 \mathrm{D}}$ would therefore be an increase of the pump spot from its present diameter of $270 \mu \mathrm{m}$ to a larger value. A complication one might, however, run into is the combined spectral and spatial labeling discussed in Ref. [14]. This labeling can be diminished by either reducing the detection bandwidth or the angle between the signal and idler beam. An alternative method to increase the Schmidt numbers is via an increase of the opening angle of the detection system. However, with the current setup we are already close to the boundary set by the thin-crystal limit.

A logical question for future research is if and how our method can be extended to an analysis of the full spatial entanglement, comprising both the azimuthal and the radial components. Recent experiments have shown how the 1D Schmidt number can be determined by measuring the twophoton visibility as a function of the transverse displacement of one beam with respect to the other [14]. We expect that a combination of 1D translation and image rotation will be sufficient to obtain enough information for a characterization of the full 2D spatial entanglement.

\section{ACKNOWLEDGMENTS}

This work has been supported by the Stichting voor Fundamenteel Onderzoek der Materie. We thank S. Verstoep for his assistance with the experiment.
[1] C. K. Hong, Z. Y. Ou, and L. Mandel, Phys. Rev. Lett. 59, 2044 (1987).

[2] P. G. Kwiat, K. Mattle, H. Weinfurter, A. Zeilinger, A. V. Sergienko, and Y. Shih, Phys. Rev. Lett. 75, 4337 (1995).

[3] N. Gisin, G. Ribordy, W. Tittel, and H. Zbinden, Rev. Mod. Phys. 74, 145 (2002).

[4] A. Mair, A. Vaziri, G. Weihs, and A. Zeilinger, Nature (London) 412, 313 (2001).

[5] S. P. Walborn, A. N. de Oliveira, S. Pádua, and C. H. Monken, Phys. Rev. Lett. 90, 143601 (2003).

[6] D. P. Caetano and P. H. Souto Ribeiro, Phys. Rev. A 68, 043806 (2003).

[7] W. A. T. Nogueira, S. P. Walborn, S. Pádua, and C. H. Monken, Phys. Rev. Lett. 92, 043602 (2004).

[8] N. K. Langford, R. B. Dalton, M. D. Harvey, J. L. O'Brien, G. J. Pryde, A. Gilchrist, S. D. Bartlett, and A. G. White, Phys. Rev. Lett. 93, 053601 (2004).

[9] S. S. R. Oemrawsingh, X. Ma, D. Voigt, A. Aiello, E. R. Eliel, G. W. 't Hooft, and J. P. Woerdman, Phys. Rev. Lett. 95, 240501 (2005).

[10] M. P. van Exter, P. S. K. Lee, S. Doesburg, and J. P. Woerdman, Opt. Express 15, 6431 (2007).

[11] M. H. Rubin, Phys. Rev. A 54, 5349 (1996).

[12] B. E. A. Saleh, A. F. Abouraddy, A. V. Sergienko, and M. C. Teich, Phys. Rev. A 62, 043816 (2000).

[13] C. H. Monken, P. H. Souto Ribeiro, and S. Pádua, Phys. Rev. A 57, 3123 (1998).

[14] P. S. K. Lee and M. P. van Exter, Phys. Rev. A 73, 063827 (2006)
[15] M. P. van Exter, A. Aiello, S. S. R. Oemrawsingh, G. Nienhuis, and J. P. Woerdman, Phys. Rev. A 74, 012309 (2006).

[16] C. K. Law and J. H. Eberly, Phys. Rev. Lett. 92, 127903 (2004).

[17] J. P. Torres, A. Alexandrescu, and L. Torner, Phys. Rev. A 68 , 050301(R) (2003).

[18] R. Grobe, K. Rzazewski, and J. H. Eberly, J. Phys. B 27, L503 (1994).

[19] A. Ekert and P. L. Knight, Am. J. Phys. 63, 415 (1995).

[20] S. Franke-Arnold, S. M. Barnett, M. J. Padgett, and L. Allen, Phys. Rev. A 65, 033823 (2002).

[21] The OAM probability $P_{l}$ and azimuthal Schmidt number $K_{\mathrm{az}}$ that we introduce are closely related to the spiral weight and spiral bandwidth introduced in Refs. [17,26]; both single out the azimuthal behavior by summing over all radial mode numbers.

[22] M. Segev, R. Solomon, and A. Yariv, Phys. Rev. Lett. 69, 590 (1992).

[23] A precise quantitative check of this value based upon the optical constants of silver is hampered by the fact that we do not know the precise thickness and porosity of the protective $\mathrm{SiO}_{2}$ coating.

[24] M. W. Beijersbergen, R. P. C. Coerwinkel, M. Kristensen, and J. P. Woerdman, Opt. Commun. 112, 321 (1994).

[25] S. S. R. Oemrawsingh, J. A. W. van Houwelingen, E. R. Eliel, J. P. Woerdman, E. J. K. Verstegen, J. G. Kloosterboer, and G. W. 't Hooft, Appl. Opt. 43, 688 (2004).

[26] L. Torner, J. P. Torres, and S. Carrasco, Opt. Express 13, 873 (2005). 